\title{
Dermatologie
}

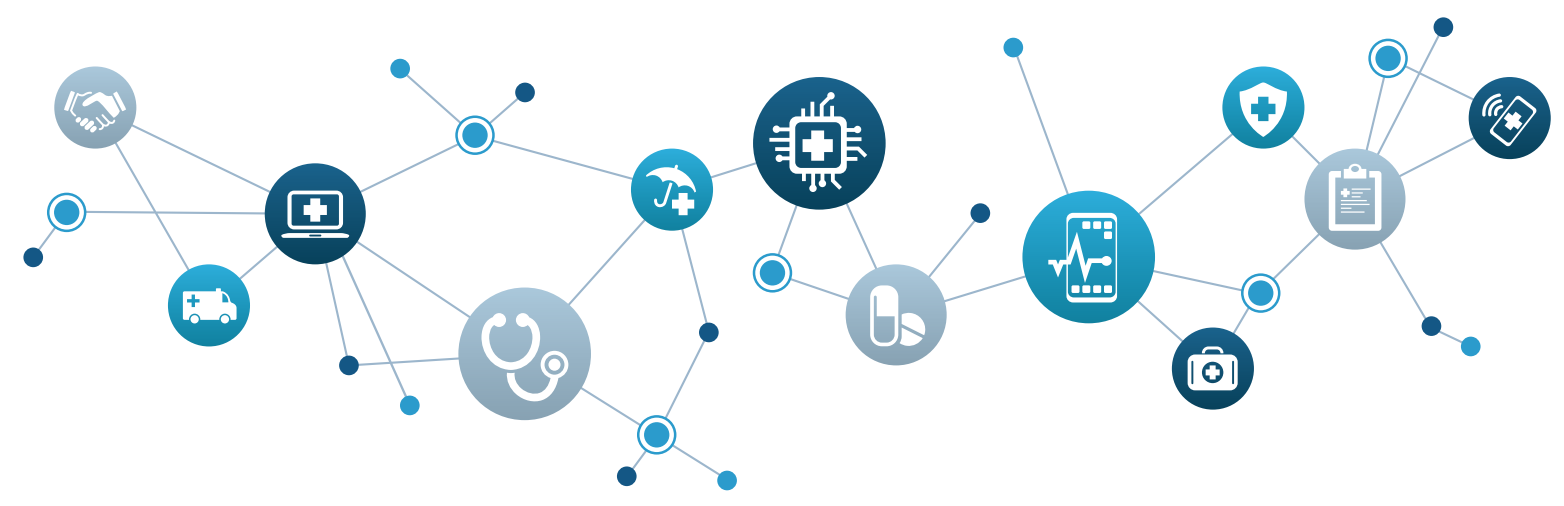

\section{Digitalisierung und Künstliche Intelligenz}

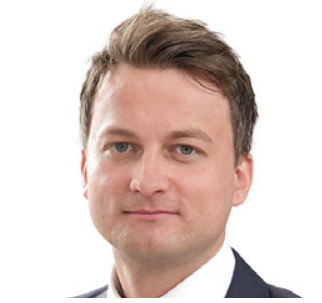

Prof. Dr. David Matusiewicz

FOM Hochschule, KCG KompetenzCentrum für Management im Gesundheits- und Sozialwesen

\section{Sozialwesen}

Wenn wir heute noch nicht einmal wissen, was die menschliche Intelligenz auszeichnet, können wir dann den Effekt der Künstlichen Intelligenz (KI) im Gesundheitswesen überhaupt einschätzen? Wird die KI langfristig den Dermatologen ersetzen oder ist diese nur ein sinnvolles Instrument des Arztes?

\section{Was ist}

Forscher sagen voraus, dass $\mathrm{KI}$ in vielen Aktivitäten in den nächsten Jahren die Leistung von Menschen übertreffen wird, wie z.B. bei der Übersetzung von Sprachen, dem Schreiben von Abituraufsätzen bzw. eines Bestsellerbuchs und schließlich in der Tätigkeit als Arzt. Rund um den Begriff KI gibt es einige Mythen, da der Begriff nicht so greifbar ist - eine Art Wolkenwissenschaft oder Marketing im Gesundheitswesen? Keineswegs. Ein Algorithmus ist wie ein Kochrezept -- gleiche Schritte führen zum gleichen Ergebnis. Auf Basis des maschinellen Lernens entsteht eine Künstliche Intelligenz, die bei ganz neuen Aufgaben (die nicht einprogrammiert wurden) nach einer Lösung sucht. Kl ist somit eine Technologie, bei der Muster in strukturierten oder unstrukturierten Daten selbstständig erkannt werden. Und das mit einer hohen Sensitivität (Anteil der tatsächlich Positiven, die korrekt als solche erkannt werden) und Spezifität (Anteil der tatsächlich Negativen, die korrekt als solche identifiziert werden).

\section{Was kommt}

Die Einsatzgebiete der KI sind in zahlreichen Fachdisziplinen der Medizin sinnvoll und werden in den nächsten Jahren deutlich ausge- baut. In der Dermatologie kann KI Hautläsionen eigenständig identifizieren und nach Relevanz ordnen. Dies ist insbesondere bei sehr großen Datenmengen oder bei einer Echtzeiteinschätzung durch den Patienten sehr sinnvoll. So hat Google auf der Entwicklerkonferenz Google I/O vor kurzem angekündigt eine KI-App mit dem Namen «Derm Assist» europaweit zu launchen, die Laien hilft, knapp 300 Hautkrankheiten zu erkennen. Nachdem der Nutzer 3 Fotos hochlädt, bekommt dieser eine Kl-gestützte Erstdiagnose. Der Grund: Google verzeichnet pro Jahr knapp 10 Milliarden Suchanfragen, die sich auf Hautprobleme beziehen. Laut einer Studie in Nature Medicine aus Jahr 2020 konnte die KI die Diagnosen mit gleicher Genauigkeit diagnostizieren wie die Dermatologen in der Kontrollgruppe [1]

\section{Was zählt}

Eine zunehmende Anzahl von Studien und Daten in der Dermatologie zeigt somit, dass KI ein sinnvolles Entscheidungsunterstützungssystem ist. So bleibt mehr Zeit für den Patienten, um zu erklären, welche Diagnostik- und Therapieempfehlungen es gibt. Die Technologie kann somit als Instrument zur Kommunikation und Entscheidungsunterstützung eine große positive Wirkung erzeugen. Die KI wird nicht so schnell den Arzt ersetzen, sie bleibt zwar nur ein Instrument, um beispielsweise Fälle vorzusortieren oder eine erste schnelle Einschätzung zu geben, wie es die Beiträge im vorliegenden Heft aus der Praxis aufzeigen. Die Restsicherheit kann heute allerdings nur der Dermatologe mit dem Auflichtmikroskop oder einer Gewebeprobe liefern. Durch die KI kann sich der Arzt auf wirklich wichtige Dinge konzentrieren: das Gespräch mit dem Patienten. Denn auch nach der Diagnostik gibt es für die Domäne der Dermatologen in der Therapie und Beratung noch genug zu tun.

Literatur Liu Y, Jain A, Eng C et al.: Nat Med 2020;26:900-908. 


\section{Künstliche Intelligenz - Maschinelles Lernen - Algorithmen Kennen Sie die Unterschiede?}

Im Zusammenhang mit der Digitalisierung werden häufig die Begriffe «künstliche Intelligenz», «maschinelles Lernen» und «Algorithmen» verwendet. Doch was verbirgt sich dahinter eigentlich? Dr. Alice Martin, Laura Siebertz, Dr. med. Estefanía Lang und Dr. med. Barbara Meyer-Lehmann bringen für uns Licht ins Dunkel.

Der Begriff der Künstlichen Intelligenz (KI) im Gesundheitswesen ist nicht neu, er wird bereits seit Jahrzehnten diskutiert. Auch wenn die $\mathrm{KI}$ immer noch mit Skepsis betrachtet wird, hält sie in immer mehr Fachgebiete und Anwendungsbereiche Einzug. Zu den ersten Fachbereichen zählten dabei die Radiologie und Kardiologie, aber auch in der Dermatologie, als visuell geprägtes Fach, wird die KI vermehrt eingesetzt. Ziel ist es, die künstliche Intelligenz (KI), oder auch die artifizielle Intelligenz (Al), in der Medizin für eine präzisere Diagnostik und eine zielgerichtete und punktgenaue Behandlung einzusetzen.

Und dabei gibt es große Unterschiede in der Programmiersprache. Man unterscheidet drei Systeme:

- Algorithmus

- Maschinelles Lernen

- Künstliche Intelligenz

In der wissenschaftlichen Literatur findet sich bislang keine exakte, allgemeingültige Definition des Begriffs «Algorithmus». Umschreiben soll der Begriff des Algorithmus jedoch eine Folge von Anweisungen, mit denen eine Lösung für eine gegebene Aufgabe gefunden werden kann. Das Lösungsverfahren ist eine Anleitung für eine Abfolge von Schritten zu der bestimmten Problemlösung, im Sinne einer «Wenn-Dann-Funktion».

Beim maschinellen Lernen durchsucht eine Software große Datenmengen und identifiziert dabei gemeinsame Merkmale, zum Beispiel Muster und Gesetzmäßigkeiten. Nach diesem Training kann das System dann unbekannte Datensätze von ähnlicher Art analysieren und Zusammenhänge erkennen, Rückschlüsse ziehen und Vorhersagen treffen. Mehrere tausend Datensätze werden für die richtige Zuordnung und Erkennung je Erkrankungsbild benötigt. Diese wiederum werden aufgeteilt in mehrere Untergruppen: eine zum Lernen (50\% des Datensatzes), eine zur Kontrolle (20\%) und eine zur Prüfung des Programms (30\%) [1].

Die komplexeste Stufe ist die Künstliche Intelligenz als zentrale Technologie der Digitalisierung. Sie ist allgemein als Überbegriff für Anwendungen zu verstehen, bei denen Maschinen die menschliche Intelligenz nachahmen, um Aufgaben durchzuführen und sich basierend auf gesammelten Daten nach und nach selbst zu verbessern. Eng verknüpft sind damit die Begriffe Algorithmus und Maschinelles Lernen, die wenigsten Programme basieren tatsächlich auf einer KI. Im Zuge der Öffentlichkeitsarbeit werden daher die
Begriffe Maschinelles Lernen oder Algorithmus häufig der KI gleichgesetzt, ohne dieser zu entsprechen. Klassische Programmiersprachen sind u.a. Python, R oder Java. Als Grundlage dienen spezialisierte Hardware und Software zum Schreiben und Erlernen des Algorithmus. Die KI könnte dazu beitragen, die überwältigende Menge an klinischen Daten, medizinischer Literatur sowie Bevölkerungs- und Nutzungsdaten sinnvoll zu nutzen, um fundierte Entscheidungen zu treffen.

\section{Anwendungsbeispiele}

\section{Google}

Die neue Google - App «Derm Assist», die bis Ende 2021 europaweit in die Google Suchmaschine integriert wird, soll Patienten und Ärzten bei der Früherkennung von Hautkrankheiten unterstützen. Nutzer können drei Fotos ihrer Haut aus verschiedenen Winkeln hochladen, Fragen zum Hauttyp und zu Symptomen beantworten und daraufhin eine Kl-gestützte Erstdiagnose erhalten. Dabei sollen von 288 Erkrankungen die wahrscheinlichsten 3 Hauterkrankungen ermittelt werden. Dabei macht Google klar, dass die App nur eine Orientierung bieten und nicht den Dermatologen ersetzen soll. Das angewandte KI-Modell wurde von der EU als medizinisches Produkt der geringsten Risikoklasse bestimmt und ist in den USA noch nicht zugelassen. In einer klinischen Studie konnte gezeigt werden, dass bei 963 Validierungsfällen das Programm sechs Dermatologen nicht unterlegen und sechs Hausärzten sowie sechs Krankenschwestern überlegen war [2]. Hier zeigt sich nun deutlich, dass zwar nicht die Expertise im Fachgebiet übertroffen wurde, aber andere Fachgebiete von solchen Fortschritten profitieren können.

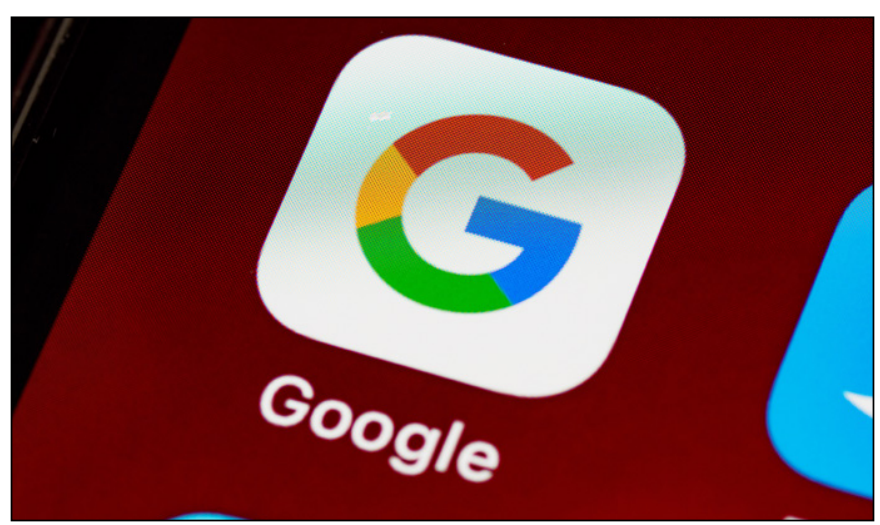

KI-gestützte Erstdiagnose: Bis Ende 2021 wird die Google-App «Derm Assist» fester Bestandteil der Suchmaschine sein. @ Brett Jordan/Unsplash

\section{Radiologie}

Ähnlich zur Dermatologie, ist die Radiologie eine bildbasierte Fachdisziplin. So wurde der Einsatz der künstlichen Intelligenz bereits deutlich früher durchgeführt und untersucht. Im Jahr 2020 wurden 
119 Softwareangebote von 55 Unternehmen ermittelt mit insgesamt 46 verschiedenen Algorithmen, die über die «Food and Drug Administration» bzw. «Conformité Européenne» eine Zulassung verfügen. Schwerpunkt der Software ist die Erkennung von Karzinom-verdächtigen Läsionen im Brust-CT und der zweidimensionalen Mammographie [3]. Insbesondere in der Frühdiagnostik und Vorsorgeuntersuchung stellt das Mammakarzinom einen großen Stellenwert dar, sodass der Einsatz vorteilhaft genutzt werden kann.

\section{Anästhesiologie}

Maschinelle Bildbeurteilungen in der Dermatologie oder Radiologie sind naheliegend und einfach im Verständnis. Aber auch andere Fachbereiche ohne Bildbeurteilungsmöglichkeiten liefern einen Zugang zu maschinellem Lernen und künstlicher Intelligenz durch die Analyse von Datensätzen. Dabei ist die künstliche Intelligenz auch in der Anästhesiologie immer weiter auf dem Vormarsch. Hier findet die Anwendung in der Überwachung der Narkosetiefe, der Anästhesiesteuerung sowie der Ereignis- und Risikovorhersage statt. Dabei stützt sich die intensivmedizinische Überwachung auf Erfahrungen der Anästhesisten, die Faktoren der Patienten und die verfügbare Medikation. Im Vergleich zur Bildanalyse sind die Prozesse teilweise komplexer und variabler aber auch die Datenmenge und Kombinationsmöglichkeiten individueller. Aus diesem Grund sind diese Möglichkeiten nur situativ und ergänzend zu betrachten [4].

\section{Vor- und Nachteile und Ausblick}

Diagnoseverfahren entsprechend der KI können Ärzte dabei unterstützen, Erkrankungen präziser und frühzeitiger zu erkennen und damit den Therapieerfolg zu verbessern. Von Patienten- als auch von Arztseite herrscht oft die Skepsis, dass durch die Anwendung der $\mathrm{KI}$ der Arztberuf überflüssig wird und die Zuwendung verschwindet. Die KI wird jedoch mehr als Entscheidungsunterstützungssystem betrachtet, bei dem das System auf Basis eigener Lernerfahrungen eine Diagnose vorschlägt. Die Programme müssen außerdem eine spezifische Prüfung durchlaufen und als Medizinprodukt klassifiziert werden. Da bei falscher Diagnose im schlimms- ten Fall auch Hautkrebs übersehen werden kann, ist der Einsatz nur nach sorgfältiger Prüfung und Durchführung von Studien möglich. Für die Überprüfung des Diagnosevorschlags ist ein behandelnder Arzt zuständig. Dabei bedeuten die Vorteile der KI vor allem, dass der Arzt sogar mehr Zeit für einen persönlichen Kontakt hat. So können diese sogar einen positiven Effekt auf die Arzt-PatientenKommunikation haben. Und auch hat der Arzt mehr Zeit für Patienten mit einer schwerwiegenden Erkrankung, da Patienten mit der Kl einfacher «kategorisiert» werden können.

Natürlich gibt es neben den Chancen auch Herausforderungen für die Patientensicherheit. Ärzte müssen lernen, mit der Zweitmeinung umzugehen. Denn der Arzt übernimmt auch hier weiter die Haftung für potenzielle Fehldiagnosen [5].

Die vielversprechenden Ansätze bedürfen allerdings weiterer Forschungen, um das Einsatzspektrum der KI-Diagnose zu erweitern und die Treffsicherheit der Ergebnisse zu erhöhen. So ist eine ausreichende Menge von Daten notwendig, dass diese als repräsentativ genug gelten können. Und noch viel wichtiger ist das Vertrauen, das erst über eine gewisse Zeitspanne von den Patienten und Ärzten aufgebaut werden kann. Die ersten Schritte sind wir bereits gegangen, aber es gibt noch viele Hürden.

\section{Literatur \\ 1. Russell S, Norvig P: New Jersey, Prentice Hall, 2009. \\ 2. Yuan Liu et. al.: nature medicine 2020; 26. \\ 3. Yasasvi Tadavarthi: Radiol Ardif Intell 2020 Nov. \\ 4. Daniel A Hashimoto: Anesthesiology 2020 Feb. \\ 5. L. Lehmann et. al.: Karger Kompass Dermatologie 2020 Nov.}

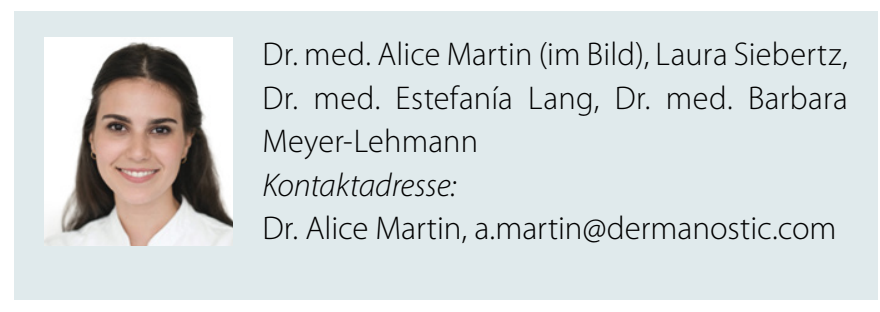




\section{$\mathrm{Kl}$ in der Medizin: Unsicherheiten in den Griff bekommen}

Künstliche Intelligenz und Maschinelles Lernen werden die Medizintechnik der nahen Zukunft prägen. Aber komplexe Verfahren des Machine Learning stellen Forscherinnen und Forscher vor ein Problem: Das Ausschließen von Fehlern vor der Inbetriebnahme autonomer Systeme sowie die zuverlässige Erkennung von Fehlern zur Laufzeit sind aufwendig und anspruchsvoll. Dies schränkt aktuell den Einsatz von Machine Learning und Künstlicher Intelligenz in sicherheitskritischen Systemen, auch in der Medizin, deutlich ein und erfordert neue Ansätze, um auch zukünftig von den Fortschritten dieser Technologie zu profitieren.

Algorithmen der Künstlichen Intelligenz (KI) werden ohne menschliches Zutun trainiert, bewertet und möglicherweise sogar ständig autonom angepasst. Aus diesem Grund werden sie oft als «Blackbox»Technologie bezeichnet. Obwohl KI flexibel ist und theoretisch mehr leisten kann als Menschen und von Menschen ausgeführte statistische Methoden, sind viele Aspekte und vor allem die Logik hinter der Entscheidungsfindung oft nicht interpretierbar. Wenn etwa ein Dermatologe eine personalisierte Bewertung durchführt und zu einer Schlussfolgerung kommt, kann diese auf der Grundlage vorhandener klinischer Beweise erklärt werden. Dagegen können zum jetzigen Zeitpunkt Entscheidungen, die von einer KI getroffen werden, nicht auf diese Weise interpretiert werden.

Dies ist eine starke Einschränkung, denn sie wirkt sich darauf aus, ob und unter welchen Bedingungen die Gesellschaft und die Aufsichtsbehörden KI in der täglichen Praxis der Medizin akzeptieren werden. Mit anderen Worten: Um Künstliche Intelligenz in solchen sicherheitskritischen Anwendungen einsetzen zu können, muss sie kontrollierbar sein, das heißt: Es muss abschätzbar sein, wie unsicher die Systemergebnisse sind. Solche Unsicherheitswerte geben Aufschluss darüber, wie zuverlässig die aktuellen Vorhersagen der $\mathrm{KI}$ sind. Zudem dienen sie als Einflussfaktoren für andere Systemkomponenten, um den aktuellen Sicherheitszustand zu bewerten und die Entscheidungen der KI abzusichern.

Die Bestimmung dieser Unsicherheit ist eine beträchtliche Herausforderung. Man kann sich viele Modelle Maschinellen Lernens (ML), insbesondere neuronale Netze, als sehr komplexe, verschachtelte Funktionen vorstellen, die zum Beispiel im Falle der Klassifizierung einem Bild Kategorien zuordnen. Beim Training solcher Modelle werden, vereinfacht dargestellt, deren Parameter so angepasst, dass die Konfidenzwerte - Maße für die Sicherheit einer Aussage - der jeweils richtigen Klassen steigen (im Gegensatz zu den falschen).

\section{Akzeptanz als entscheidendes Kriterium}

Was heißt das für die Medizin im Allgemeinen und die Dermatologie im Speziellen? Eine ordnungsgemäße Anamnese, gefolgt von einer Untersuchung in einer gut ausgeleuchteten Umgebung, bei der Texturen beurteilt und spezifische Anzeichen für bestimmte Läsionen festgestellt werden, ergänzt durch zusätzliche Untersuchungen und/ oder Bildauswertungen oder eine Biopsie, ist ein Standardverfahren zur Erstellung einer Diagnose in der Dermatologie. Darüber hinaus wird anerkannt, dass einige Diagnosen klinisch sind, während andere ausschließlich auf histologischen Befunden oder einer Kombination aus beiden beruhen. Dieser ganzheitliche Ansatz kann nicht vollständig durch Computerprogramme ersetzt werden, und dies wird als eines der wichtigsten Hindernisse für die Einführung der KI angesehen. Viele Patientinnen und Patienten möchten auch einfach einen Arzt sehen, der sich für sie einsetzt und mit dem sie gemeinsam die Behandlung angehen, anstatt sich möglicherweise mit einem isolier-

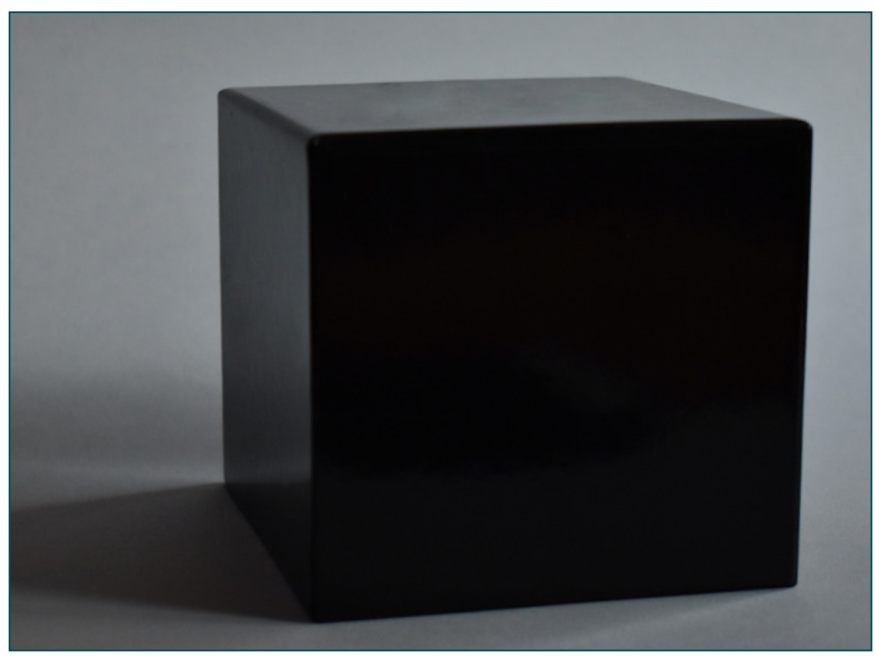

Blackbox KI: Viele Aspekte und vor allem die Logik hinter der Entscheidungsfindung einer KI sind oft nicht interpretierbar. Foto: Tommy Diner/Unsplash

ten computergestützten Programm zufriedenzugeben.

\section{Das Fraunhofer IKS macht KI selbstkritischer}

Aber zurück zur Technologie. Es kann auch ein Problem sein, dass viele etablierte Methoden des Maschinellen Lernens von bestimmten Einzelwerten für Parameter ausgehen statt von deren Verteilungen. Dies ist suboptimal, wenn ein einziges, starres Beispiel reale Muster und Zusammenhänge nur unzureichend beschreibt. Es erhöht das Risiko, dass die Modelle in ungünstigen Fällen irreführend sind, und weist auf eine weitere Ursache für unzuverlässige Konfidenzwerte hin: Modelle werden auf Datensätzen trainiert, die nicht alle relevanten Zustände repräsentieren (zum Beispiel Hautfarben, Beleuchtungsunterschiede, Fokus etc.) und kommen so zu falschen Ergebnissen.

Um diese Fehler zu vermeiden, entwickelt das Fraunhofer-Institut für Kognitive Systeme IKS neuartige Methoden: Damit lassen sich solchen Fällen höhere Unsicherheitswerte zuweisen, die stark von den gelernten Konzepten aus dem Trainingsdatensatz abweichen. So kann das Modell besser anzeigen, wann es in seiner Vorhersage unsicher ist. Ein Forschungsschwerpunkt ist die sichere prädiktive Modellierung für Anwendungen im Gesundheitswesen: ein multidisziplinärer Ansatz für Kl, der Maschinelles Lernen, 
kausale Inferenz und Optimierung im Gesundheitswesen kombiniert. In diesen angewandten Projekten entwickelt und überführt das Fraunhofer IKS neuartige ML- und Kausalschlussverfahren aus der Theorie in die Praxis, um das Auftreten zukünftiger unerwünschter Ereignisse, Komplikationen und Ergebnisse unter Verwendung früherer Informationen über den Krankheitsverlauf von Patienten vorherzusagen.

Dabei werden Fragen beantwortet wie:

- Wird dieser Patient aufgrund seiner Vorgeschichte eine bestimmte Erkrankung entwickeln?

Es geht auch darum, Patientenkategorien mit erhöhtem Risiko zu identifizieren, zum Beispiel das Komplikationsrisiko bestimmter Behandlungen für Patienten zu bestimmen. Ferner gilt es, direkte und indirekte Einflüsse von Behandlungsentscheidungen auf das Patientenergebnis zu bewerten, also eine Mediationsanalyse vorzunehmen. Dazu gehört schließlich auch die Entwicklung von Techniken zur Bewertung des Einflusses alternativer Behand- lungsentscheidungen auf das Patientenergebnis, also eine kontrafaktische Analyse zur Beantwortung von Fragen wie:

- Hätte dieser Patient ein anderes Ergebnis gehabt, wenn die Ärzte in der Vergangenheit eine andere Behandlung durchgeführt hätten?

In all diesen Bereichen ist die Forschung des Fraunhofer IKS besonders daran interessiert, die Unsicherheit von Beobachtungen und Entscheidungen sowohl für Menschen als auch für KI zu quantifizieren, robuste Modelle in Anwesenheit von nicht-zufällig fehlenden Daten zu entwerfen und zuverlässige Validierungsmetriken zu entwickeln, die sich an klinischen Bedürfnissen orientieren.

Dr. Narges Ahmidi, Leiterin der Abteilung Reasoned Al Decisions am Fraunhofer-Institut für Kognitive Systeme IKS in München Kontaktadresse:

Hans-Thomas Hengl, hans-thomas.hengl@iks.fraunhofer.de

\section{Datenschutz: Herausforderungen und Grenzen des Trainings künstlicher Intelligenzen}

Eine künstliche Intelligenz (KI), die die Diagnosestellung beschleunigt, Ärztinnen und Ärzte unterstützt und Diagnosen zuverlässiger macht? Klingt gut. Aber eine KI muss trainiertwerden. Wie die dafür benötigten Daten erhoben und verarbeitet werden können, ohne dass der Datenschutz verletzt wird, erklären uns Dr. Werner Schäfke-Zell und Marius Post, Caladan GmbH, aus dem Arbeitskreis Datenschutz und -sicherheit des Spitzenverbandes digitale Gesundheitsversorgung e.V.

Wie wichtig das Thema «künstliche Intelligenz» ist, und wie weit oben es auf der politischen Agenda steht als Möglichkeit für breiten wirtschaftlichen und gesellschaftlichen Fortschritt, zeigt sich in der Begründung für den Vorschlag des Europäischen Parlaments für ein «Gesetz über künstliche Intelligenz»:

«Künstliche Intelligenz (KI) bezeichnet eine Reihe von Technologien, die sich rasant entwickeln und einen vielfältigen Nutzen für Wirtschaft und Gesellschaft über das gesamte Spektrum industrieller und gesellschaftlicher Aktivitäten hinweg hervorbringen können. Der Einsatz künstlicher Intelligenz zur Verbesserung von Prognosen, zur Optimierung von Abläufen und der Ressourcenzuweisung sowie zur Personalisierung der Diensteerbringung kann für die Gesellschaft und die Umwelt von Nutzen sein und Unternehmen sowie der europäischen Wirtschaft Wettbewerbsvorteile verschaffen. Bedarf besteht insbesondere in Sektoren, von denen eine große Wirkung ausgeht, wie Klimaschutz, Umwelt und Gesundheit, öffentlicher Sektor, Finanzen, Mobilität, Inneres und Landwirtschaft.» (1)
Im Gesundheitssektor rührt die Relevanz der Nutzung künstlicher Intelligenz insbesondere von der dünnen Facharztdecke. Die Nutzung künstlicher Intelligenz verspricht, die Effektivität der Diagnosestellung zu steigern. Daneben gibt es noch weitere denkbare Nutzen wie die Möglichkeit, dass künstliche Intelligenz auch die Zuverlässigkeit von Diagnosen erhöhen könnte.

Regulatorisch gesehen, bewegt sich KI in der Dermatologie in der Schnittmenge zwischen Medizinprodukt, Telemedizin, Datenschutz und demnächst zusätzlich der Regulierung künstlicher Intelligenzen.

\section{Welche Bedingungen gibt es für den Einsatz von KI und Deep Learning?}

Aus datenschutzrechtlicher Sicht ist der Vorgang der Entwicklung einer Kl, beispielsweise durch Deep Learning, und der Vorgang der Nutzung einer solchen $\mathrm{KI}$ in der Behandlung von Patienten zu trennen.

Die Entwicklung einer KI bedarf eines realen Trainingsdatensatzes. Findet die Entwicklung im Rahmen wissenschaftlicher Forschung statt, halten die Datenschutz-Grundverordnung (DSGVO) sowie das Bundesdatenschutzgesetz (BDSG) spezielle Erlaubnistatbestände bereit, Gesundheitsdaten gegebenenfalls auch ohne Einwilligung der Betroffenen für das Training einer KI zu nutzen. Handelt es sich bei den Daten um Patientendaten, die im Rahmen der ärztlichen Behandlung erhoben wurden, muss jedoch immer auch eine Entbindung des betreffenden Arztes von der Schweigepflicht vorliegen, die die Übermittlung der Daten an eine Forschungseinrichtung oder eine andere Stelle erlaubt, die das KI-Training vornimmt.

Falls die Entwicklung einer KI vornehmlich kommerziellen Interessen dient, geht die Kommentarliteratur davon aus, dass sie nicht unter das 
Privileg wissenschaftlicher Forschung fällt. In diesen Fällen ist es ratsam, Einwilligungen der Betroffenen einzuholen, dass ihre Daten zu Zwecken des KI-Trainings erhoben werden. Alternativ kann im Rahmen einer Interessenabwägung eine zweckändernde Verarbeitung legitimiert werden. Auch diesen Weg erlauben DSGVO und BDSG. Die Hürden sind hier jedoch hoch, und es muss ein einfacher Weg für die Betroffenen geboten werden, der Verarbeitung zu widersprechen. Die Anwendung einer KI für Behandlungszwecke muss vor allem den Anforderungen der DSGVO an automatisierte Entscheidungen im Einzelfall genügen. Wie so häufig im Datenschutz - und im Patientenrecht - sind Informiertheit und Kontrolle der betroffenen Patienten sowie angemessene Sicherheitsvorkehrungen maßgeblich für die Rechtmäßigkeit des Einsatzes einer KI für Diagnose und Behandlung. Der Patient muss informiert sein, dass eine KI eingesetzt wird und hat das Recht, das Eingreifen einer Person - z. B. des behandelnden Dermatologen - zu erwirken. Eine Einwilligung in den Einsatz einer KI bei den Patienten einzuholen ist möglich, aber rechtlich nicht zwingend erforderlich, sofern der Einsatz aus dem Behandlungsvertrag mit dem Arzt hervorgeht.

\section{Welche Daten werden erhoben?}

Welche Daten erhoben werden können und dürfen richtet sich zunächst nach dem Grundsatz der Datenminimierung. Es dürfen diejenigen Daten erhoben werden, die für die Erreichung des Zwecks angemessen, erheblich und auf das notwendige Maß beschränkt sind. Nutzt man Daten, die sich aus der Behandlung von Patienten ergeben, kann man alle die Daten aus der Patientenakte nutzen, die für die gewählte Kl und die Aufgaben, die sie erfüllen können soll, notwendig und erheblich sind. Es ist möglich, darüberhinausgehende Daten zu erheben, sofern eine Interessenabwägung dies unterstützt, da es möglich ist, Daten zu erheben, die für die Durchführung eines (Behandlungs)Vertrags nicht erforderlich sind, aber dennoch eine wichtige, unterstützende Rolle für dessen Durchführung spielen können.

\section{Wie werden diese Daten verarbeitet?}

Beim Deep Learning als einer Form des maschinellen Lernens werden Daten durch ein künstliches neuronales Netz in mehreren Schritten auf immer weiter steigenden Abstraktionsebenen analysiert. Die Ausgabe des Systems ist üblicherweise eine Klassifizierung eines Inputs in vorgegebene Kategorien. Was genau zwischen Eingabe und Ausgabe passiert, ist für Menschen nicht beobachtbar, nur die Genauigkeit der Klassifizierungen, die das künstliche neuronale Netz vornimmt, kann evaluiert werden. Viel hängt also an gut gewählten Trainingsdaten. Nicht umsonst sind unbeabsichtigte Vorurteile, die künstliche Neuronale Netzwerke entwickeln, ein häufiges Thema. Denkt man sich einen dermatologischen Trainingsdatensatz, der von Patienten mit vornehmlich heller Hautfarbe stammt, kann die Zuverlässigkeit der Anwendung bei Patienten mit dunkler Hautfarbe möglicherweise geringer sein und umgekehrt, da die KI die Hautfarbe nicht von der Hautveränderung abstrahiert hat.

\section{Von wem werden diese Daten verarbeitet?}

Die Problematik eines gut zusammengesetzten Trainingsdatensatzes verdeutlicht die Herausforderungen und Grenzen des Trainings

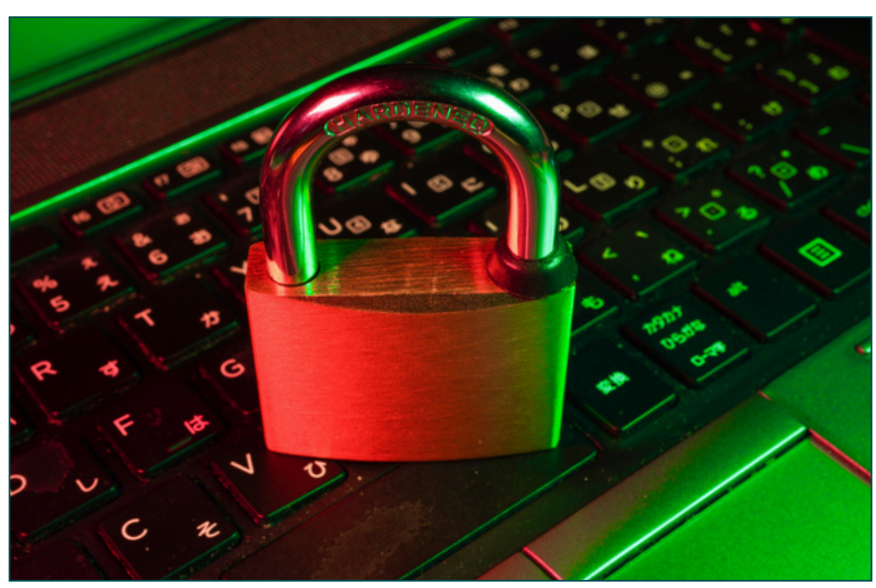

Datensätze mit personenbezogenen Daten genießen, berechtigterweise, einen besonderen Schutz. Foto: FLY:D/Unsplash

künstlicher Intelligenzen. KI-Training wird deswegen häufig von spezialisierten privaten Dienstleistern oder universitärer Forschungsgruppen vorgenommen. Aus Sicht des Datenschutzes macht es keinen Unterschied, ob der oder die Dermatologin selbst, eine ihrer Mitarbeiterinnen oder ein Dienstleistungsunternehmen das KI-Training vornimmt. Alle beteiligten Akteure haben für ein angemessenes Schutzniveau der Daten bei jedem Schritt ihrer Verarbeitung Sorge zu tragen und haften für die fahrlässige oder vorsätzliche Verletzung des Datenschutzes.

Eine offene, «freie» Forschung ist mit Gesundheitsdaten kaum zu betreiben. Es bestehen berechtigterweise sehr hohe Hürden für die Veröffentlichung von Datensätzen mit personenbezogenen Daten, ob nun für Zwecke der wissenschaftlichen Forschung oder für die vornehmlich gewinnorientierte Produktentwicklung. Der nahezu einzige legale Weg ist die Anonymisierung der Daten. Eine datenschutzkonforme Anonymisierung ist jedoch weit schwieriger als man landläufig annimmt, weil eine auch nur indirekte Zuordnung von Individuen zu einem Datensatz über Daten wie Geburtstag, Postleitzahl und Geschlecht nicht möglich sein darf. Bei Bilddaten, wie sie die Dermatologie benötigt, ist ein KI-Training auf der Grundlage geschwärzter Bilder nicht möglich und Anonymisierung nicht gangbar. Gleichzeitig ist eine Einwilligung für die Veröffentlichung der Daten kaum möglich, da Einwilligungen nur für spezifischere Zwecke gegeben werden dürfen als für die Forschung allgemein durch wen auch immer.

\section{Literatur}

Europäisches Parlament (21.04.2021): Gesetz über künstliche Intelligenz, vom Gesetzesvorschlag. Online verfügbar unter https://eur-lex.europa.eu/legal-content/DE/TXT/HTML/?uri= CELEX:52021PC0206erfrom=EN, zuletzt geprüft am 16.09.2021.

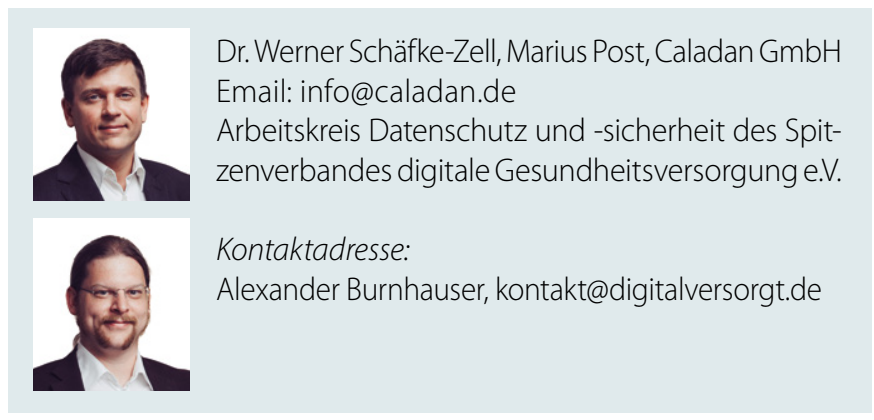




\section{Wearables im Einsatz bei atopischem Ekzem - ein neuer präventiver Ansatz}

Hohe Kosten, Zeitmangel oder Incompliance der Patienten: Die Therapie und Betreuung von Neurodermitis-Betroffenen lässt sich nicht immer perfekt umsetzen. Hospitalisierungsraten, Patientenunzufriedenheit und Frustration des Arztes steigen. Wie lässt sich dies verhindern? Am Klinikum rechts der Isar der TU München wird daran geforscht, wie Wearables und Kl einen Beitrag leisten können.
16.30 Uhr, Dermatologieambulanz, Klinikum rechts der Isar, München: Frau H., eine 50-jährige Patientin mit diagnostiziertem atopischem Ekzem sitzt im Wartezimmer der Hautklinik und wartet darauf, von ihrem Arzt aufgerufen zu werden. Seit Jahren leidet sie unter schwerer Neurodermitis und muss regelmäßig zur Therapieanpassung und Kontrolle in die Ambulanz der Klinik kommen. So wie Frau H. geht es vielen Patienten, die unter einer schweren Form der chronischen Erkrankung leiden. Nicht umsonst gilt die atopische Dermatitis als eine der am stärksten belastenden Krankheiten weltweit: getriggert durch multiple Faktoren wie beispielsweise Stress oder bestimmte Allergene kann es zur starken Exazerbation der Haut kommen [1-3]. Komplikationen wie Ekzema herpeticatum oder bakterielle Superinfektionen mit Staphylococcus aureus sind gefürchtete Folgen [3-5]. Wie das TREATgermany-Register, ein wissenschaftlich geleitetes prospektives Forschungsregister zur Beobachtung von Patienten mit moderater bis schwerer Neurodermitis, feststellte, konnte bei betroffenen Patienten nicht selten eine langfristige Einschränkung der Arbeitsfähigkeit und Lebensqualität bewiesen werden, die neben Stigmatisierung häufig auf Superinfektionen zurückzuführen ist [6].

Eine individuelle personalisierte Langzeitpflege ist unabdingbar, da die Haut täglichen Schwankungen und multiplen Einflussfaktoren unterliegt [1]. Und besonders die Feuchtigkeitspflege gilt als «bedrock» in der Therapie des atopischen Ekzems, so auch bei allen internationalen Leitlinien [7]

\section{Neue «homebased sensor technology» soll Patienten mit chronischem AE helfen}

Ein neuer präventiver Ansatz wird am Klinikum rechts der Isar der Technischen Universität München verfolgt. Eine Forschungsgruppe der Klinikund Poliklinikfür Dermatologie und Allergologie entwickelt derzeit in Kooperation mit den beiden Unternehmen iSYS Software $\mathrm{GmbH}$ und Eltroplan $\mathrm{GmbH}$ einen miniaturisierten Sensor in Form eines Armbandes, mit welchem eine Echtzeitmessung von für Neurodermitis relevanten Hautparametern möglich sein wird*. Dabei ist iSYS in der Entwicklung der Software federführend, Eltroplan ist für die hardware-technische Entwicklung des Wearables verantwortlich. Eingeschlossen werden sollen Parameter wie beispielsweise der transepidermale Wasserverlust (TEWL). «Die Tewamatrie** $\{. .$.$\} ist$ ein für klinische Studien wichtiger objektiver Messparameter und kann z. B. bei unzureichendem therapeutischem Ansprechen und schwierigen Verläufen hinzugezogen werden» (100 \% Konsens), so Wollenberg et. al in deren Positionspapier zu Diagnostik und Therapie von Xerosis cutis. Durch die Messung dieser Parameter können der Hautzustand und der Therapierfolg möglichst objektiv beurteilt werden, was ein Mehrgewinn für die sonst meist nur klinische Blickdiagnostik der Neurodermitis ist, vor allem für die verlaufsbeurteilung [1].

\section{Feedbackkontrolle durch den Patienten selbst im Alltag mit App} Über eine eigens für den Sensor entwickelte App können die Patienten jederzeit eine Rückmeldung über den Zustand ihrer Haut bekommen sowie Beschwerden und Hautbefunde schriftlich und mittels Fotofunktion dokumentieren. Doch nicht nur der Patient selbst profitiert davon: Neben der selbstständigen Hautkontrolle des Patienten zu Hause kann der Arzt bei Konsultationen den Verlauf der Parameter analysieren und somit den Hautzustand besser einschätzen. Außerdem können relevante äußere Einflussfaktoren erfasst und eingeschätzt werden, denn auch die Messung exogener Umweltfaktoren ist integriert. So können beispielsweise der Einfluss von Kälte, niedriger Luftfeuchtigkeit oder starker Sonneneinstrahlung als bekannte Risikofaktoren für Xerosis cutis so miterfasst werden [1].

\section{Objektive Messung des Stresslevels soll neue Rückschlüsse auf den Hautzustand erzielen}

Als bis jetzt in der klinischen Diagnostik des atopischen Ekzems standardmäßig noch nicht miterfasster Wert soll auch die elektrodermale Aktivität, kurz EDA, als Messparameter im Sensor integriert werden. Durch die Messung elektrischer Potenziale kann mit dem EDA der Widerstand der Haut ermittelt werden. Dieser steht in Zusammenhang mit dem Sympathikotonus und damit auch mit der Aktivierung von Schweißdrüsen. Dies wiederum korreliert mit dem Stresslevel [9]. Es ist kein Geheimnis, dass Stress Exazerbationen der Haut fördert und damit den Zustand eines atopischen Ekzems massiv verschlechtern kann [10]. Leider ist im Nachhinein der Auslöser der Hautverschlechterung oft nicht mehr nachvollziehbar. Mit Erfassung aller genannten Parameter inklusive des Stresslevels könnte sich dies mit dem miniaturisierten Sensor nun ändern. Es gibt bereits einige weitere Studien zum Einsatz des EDAs in anderen medizinischen Fachbereichen. Beispiele sind das Tracking des präoperativen Stresslevels bei Patienten im OP oder der diagnostische Einsatz in der Psychiatrie bei schweren Depressionen, wo EDA als quantitativer Marker dient $[9,11]$.

\section{Werden Wearables das Gesundheitssystem im 21. Jahrhun- dert revolutionieren?}

Der Einsatz des EDAs in Form von miniaturisierten Sensoren ist nur ein Beispiel für den anhaltenden Trend von Wearables. Doch was genau definiert ein Wearable? Damit gemeint sind elektronische Messgeräte, die eng am Körper getragen werden und medizinisch 


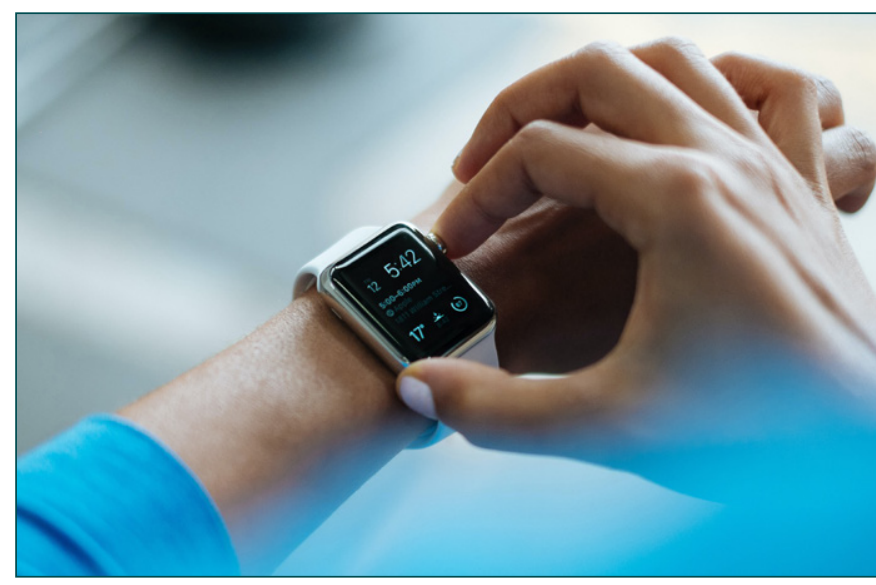

Mehr als Smartwatch: Wearables sind elektronische Messgeräte, die eng am Körper getragen werden und medizinisch relevante Parameter erfassen können Foto Luke Chesser/Unsplash

relevante Parameter erfassen können [12]. Wie eine Prognose des Marktforschungsinstitutes «Yole Développement» in Lyon zeigte, soll sich die Anzahl der Wearables innerhalb der Jahre 2016 bis 2022 mehr als verdreifacht haben. Dies zeigt den klaren Zukunftstrend hin zur Wearable Technologie [13].

Auch durch die Corona-Pandemie hat das Thema Wearables an Aufmerksamkeit gewonnen, wie eine aktuelle Trendanalyse des Fraunhofer Institutes ergab. Home-Monitoring von Vitalparametern erspart Zeit, Kosten und verringert das Infektionsrisiko. Hinzu kommt das Potenzial von Künstlicher Intelligenz (KI): Mit Hilfe von KI können durch Analyse bestimmter Parameterkonstellationen Vorhersagen getroffen werden, wann das Risiko für bestimmte Folgen erhöht ist. So im Falle vom Covid-19 Monitoring beispielsweise die Gefahr der Dekompensation bei Herzinsuffizienz [14, 15].

«Wearable Health Devices (WHDs) helfen den Menschen zunehmend dabei, ihren Gesundheitszustand besser zu überwachen, und zwar sowohl auf Aktivitäts-/Fitnessebene für die Selbstüberwachung als auch auf medizinischer Ebene, indem sie den Ärzten mehr Daten liefern, die eine frühere Diagnose und eine gezieltere Behandlung ermöglichen» [16].

So lässt sich als Fazit zum sensorbasierten Monitoring der Neurodermitis sowie auch zu Wearables allgemein sagen: Ein hoffentlich vielversprechender Ansatz, von welchem Patient, Arzt und Gesundheitssystem profitieren können.

\section{Projektträger und Projektpartner}

- Zentrales Innovationsprogramm Mittelstand

- Gefördert durch das Bundesministerium für Wirtschaft und

Energie aufgrund eines Beschlusses des Deutschen Bundestages

- Eltroplan Engineering GmbH

- Klinikum rechts der Isar Technische Universität München

- iSYS Software GmbH
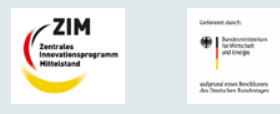

\section{'EItroplan}

* Anmerkung: Es handelt sich hierbei um ein FuE-Kooperationsprojekt der AiF Projekt $\mathrm{GmbH}$, welches vom Bundesministerium für Wirtschaft und Energie aufgrund eines Beschlusses des Deutschen Bundestages gefördert wird und im Rahmen des Programmes «Zentrales Innovationsprogramm Mittelstand» (ZIM) läuft.

**Anmerkung: Die Tewametrie ist das Standardverfahren, welches zur Beurteilung des transepidermalen Wasserverlustes eingesetzt wird. Standardmessgeräte zur Durchführung sind meist Tewameter TM210 oder TM300 [8].

\section{Literatur}

Die Literatur ist unter www.karger.com/doi/10.1159/000520398 abrufbar.

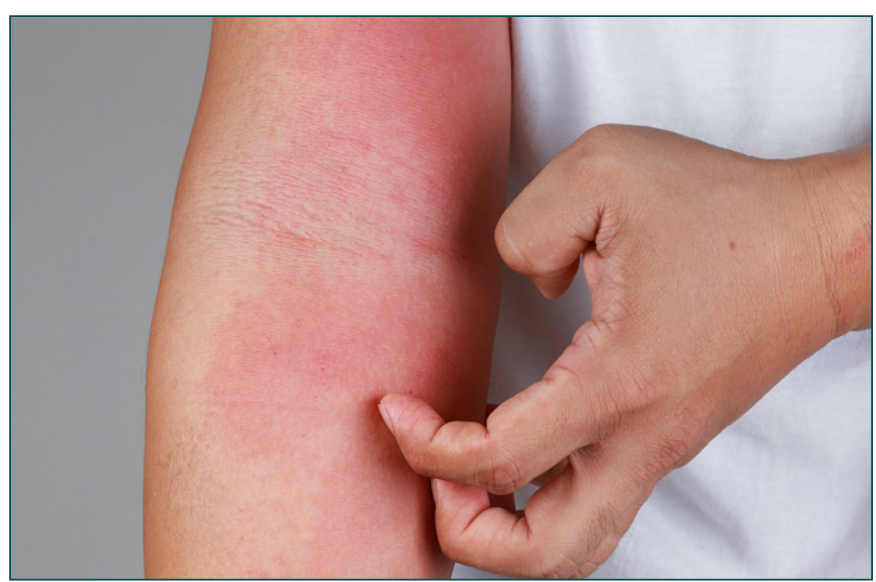

Für Neurodermitis-Betroffene ist eine individuelle personalisierte Langzeitpflege unabdingbar

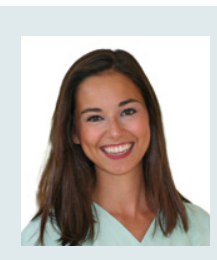

Charlotte Kiani' (im Bild), Marc Ponschab², Andreas Frey², Rainer Baumgärtner ${ }^{2}$, Hendrik Kaltenbach ${ }^{3}$, Claus Sembach ${ }^{3}$, Jan Kokert ${ }^{3}$, Axel Frank ${ }^{3}$, Alexander Zink ${ }^{1}$

\section{Affiliation:}

${ }^{1}$ Klinik und Poliklinik für Dermatologie und Allergologie am Biederstein, Fakultät für Medizin, Technische Universität München, München, Deutschland

2 Firma iSYS Software GmbH, Softwareentwickler in München, Deutschland

${ }^{3}$ Firma Eltroplan Engineering $\mathrm{GmbH}$, Hersteller elektronischer Geräte in Endingen am Kaiserstuhl, Deutschland Korrespondenzadresse:

Priv.-Doz. Dr. Dr. med. Alexander Zink, alexander.zink@tum.de 


\section{KI im Einsatz: Hautkrebsprävention per Smartphone-App «SkinScreener»}

\begin{abstract}
Mit der Entwicklung der Smartphone-App SkinScreener stellt das Start-up medaia (=MEDical Artificial Intelligence Applications) aus Österreich/Graz ein auf künstliche Intelligenz (KI) basiertes und CEzertifiziertes Medizinprodukt für die Prävention von Hautkrebs bereit. Anhand eines per Handy erstellten Kamerabildes der verdächtigen Hautläsion analysiert und berechnet der klinisch erprobte Algorithmus mit einer Genauigkeitsrate von 95\% das Risiko einer Bösartigkeit.
\end{abstract}

\section{Erkennt Melanome und andere Hautkrebs}

Durch das Zusammenspiel zweier neuronaler Netzwerke sowie einer großen Anzahl von mehreren Schichten Neuronen werden von der App alle medizinisch relevanten pigmentierten sowie nicht-pigmentierten Hauttumore erkannt. Eines der beiden neuronalen Netzwerke stellt einen klassischen Bild-Klassifikationsansatz (Analyse-Algorithmus) dar, das andere setzt auf regionenbasierte Objektdetektion (Erkennungs-Algorithmus). Mit diesem KI-Ansatz identifiziert SkinScreener alle Anzeichen von Krebsvorstufen wie aktinische Keratose und dysplastische Nävi sowie Strukturelemente bösartiger Läsionen wie Plattenepithelkarzinom, Melanom, Basaliom und Morbus Bowen. Die Anwendung ist für erwachsene Personen mit Fitzpatrick-Hauttyp I (Rothaarig) bis Typ IV (Dunkelbraun) erprobt. Der User erhält in Echtzeit die Bewertung (geringes, mittleres, hohes Risiko) über ein leicht verständliches 3-färbiges Ampelsystem. Die App stellt somit eine digitale Ergänzung zur regelmäßigen dermatologischen Untersuchung dar. Im Rahmen einer klinischen Studie an der Medizinischen Universität Graz wurden 1242 Hautläsionen von 350 Patienten gescannt - die Diagnose wurde von mindestens 3 Dermatologen pro Scan gestellt. Die Ergebnisse bei der Risikobewertung von bösartigen Hautläsionen durch das selbstlernende algorithmische System waren beeindruckend. Die Empfindlichkeit und Spezifität des Erkennungs-Algorithmus betrug 96,4\% (Cl 93,94-98,85) und 94,85\% (Cl 92,46 - 97,23), vom Analyse-Algorithmus wurde eine Sensitivität von 95,35\% (Cl 93,45 - 97,25) und eine Spezifität von 90,32\% (Cl 88,1 - 92,54) erzielt. Laut einer aktuellen Untersuchung (Liu, Y., Jain, A., Eng, C. et al. Nat Med 26, 900-908 (2020)) liegt die Diagnosegenauigkeit von Hausärzten in den USA bei nur 24 -70 \% im Vergleich zu 77 - 96 \% für Dermatologen. Die KI übertrifft somit die Genauigkeit von Hausärzten und ist mit der von Dermatologen vergleichbar.

\section{Zukunftstechnologie, die Heilungschancen erhöht}

Das Gesundheitswesen befindet sich in einer digitalen Transformation. Der verstärkte Einsatz von KI-Systemen für medizinische Anwendungen wie SkinScreener wird schon in naher Zukunft wesentlich dazu beitragen, die individuelle Gesundheit und menschlich gerechte Versorgung unabhängig von der lokalen Infrastruktur zu verbessern. Im konkreten Fall eröffnet die weltweite Verbreitung von Smartphones die Chance mehr Patienten früher zum Arztbesuch zu bewegen folglich die Früherkennungsrate von Hautkrebs und somit die Hei-

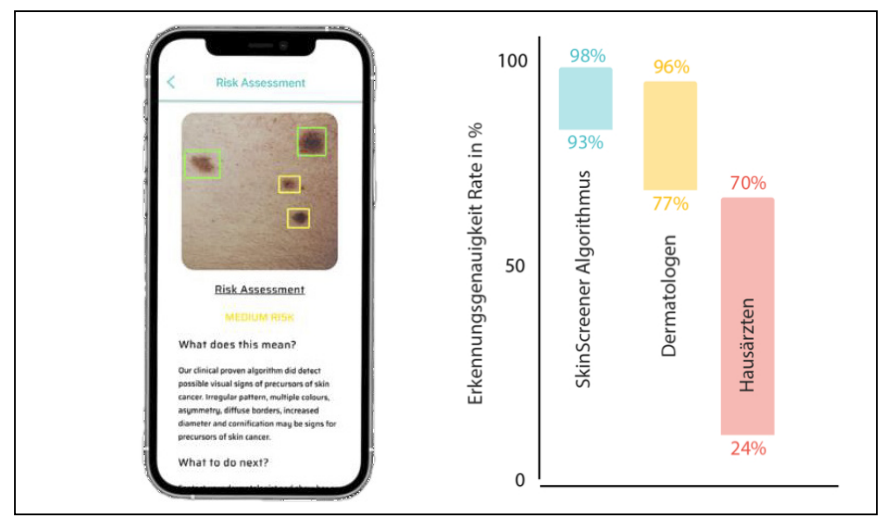

SkinScreener: Eine KI wertet die per Smartphone-Kamera fotografierten Hautläsionen aus. Untersuchungen in den USA zeigen: Die Diagnosegenauigkeit von $\mathrm{KI}$ und Dermatologen ist vergleichbar

lungsaussicht von Millionen von Menschen zu steigern. Mit KI-basierter Technologie lassen sich Folgeschäden besser vermeiden, komplexen Entscheidungen und Diagnosen leichter treffen und durch eine gezielte frühzeitige Therapie Gesundheitskosten langfristig senken.

\section{EU-weit zertifiziertes Medizinprodukt}

Dem jungen und innovativen medaia-Team, die Spezialisierungen reichen von Neurowissenschaften über Dermatologie bis hin zu biomedizinischer Technik, ist es im Vorjahr zudem gelungen, für SkinScreener die CE-Zulassung als Medizinprodukt zu erhalten. Derzeit läuft das Prüfungsverfahren zur ersten EU-weiten Kl-Zertifizierung als Medizinprodukt der Klasse CE-lla. Bisher steht die App in Österreich und Deutschland sowie in Großbritannien und Irland in den Stores von Apple als auch Google zum Download zur Verfügung. Allein an den aktuell schon über 35000 Nutzern lässt sich das große Interesse und Wachstumspotential erkennen.

KI-Systeme werden Ärzte nicht ersetzen, sondern bei repetitiven Aufgaben entlasten. Sie stellen eine nützliche Erweiterung und Entscheidungshilfe der klinischen Praxis dar. Eine schnellere Entscheidungsfindung bedeutet mehr Zeit für Patienten und Arzt. Neben der Risikostratifizierung zeigt die in der App integrierte Funktion «Dermatologe in der Nähe» einen Dermatologen in unmittelbarer Umgebung an, der im Falle einer gesundheitsgefährdenden Bewertung der gescannten Hautläsion kontaktiert werden kann. Als weiteren Hinweis für den Nutzer wird der aktuellen UV-Index in Abhängigkeit vom Standort angezeigt, um auf eine hohe Sonnen- oder UVBelastung aufmerksam zu machen.

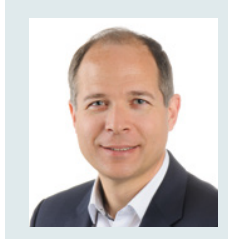

Dr. Michael Tripolt, Dermatologe und Gründer Kontakt: office@medaia.at Weitere Infos unter: www.skinscreener.com www.medaia.at

Foto: Werner Stieber 


\section{Ohne Dermatologen geht es nicht}

Die Dermatologie ist ein visuelles Fach. Damit bietet sich eine digitale Diagnosestellung ebenso an wie eine telemedizinische per App. Dabei kann vermehrt künstliche Intelligenz (KI) eingesetzt werden, um Diagnosen sicherer zu machen. Das Webinar «Karger Round Table: Digitalisierung in der Dermatologie» thematisierte dieses weite Feld Anfang Oktober 2021.

Die niedergelassene Dermatologin Dr. med. Estefania Lang, Düsseldorf, ist Mitbegründerin der teledermatologischen Praxis dermanostic. Per App sind Konsultationen des Hautarztes möglich. Nachdem ein Patient die App von dermanostic heruntergeladen und eine Registrierung absolviert hat, sollten 3 Bilder mit der App von dem betroffenen Hautareal aufgenommen werden.

Zusammen mit einem kurzen Fragebogen werden diese Dokumente dann an dermanostic geschickt. Eine Diagnose und gegebenenfalls ein Therapievorschlag sollte innerhalb von 24 Stunden dem Patienten vorliegen. Diese Form der Behandlung kostet 25 Euro. Hinter dermanostic stehen mehrere Fachärzte für Dermatologie, welche die eingehenden Informationen jeweils persönlich beurteilen und diagnostisch tätig werden.

«83\% der Patienten benötigen danach keinen weiteren Hautarztbesuch», erklärt Dr. Lang. Die Patienten erhalten einen ausführlichen Arztbrief zu Ihrer Hautveränderung, worin die Erkrankung und Therapieempfehlung präzise und verständlich beschrieben ist. Wenn nötig erhält der Patient ein Privatrezept. Bei den übrigen 17\% waren eine weiterführende Laboruntersuchung, ein chirurgisch-diagnostisches Vorgehen oder die Einleitung einer Photooder Systemtherapie indiziert, berichtete Dr. Lang.

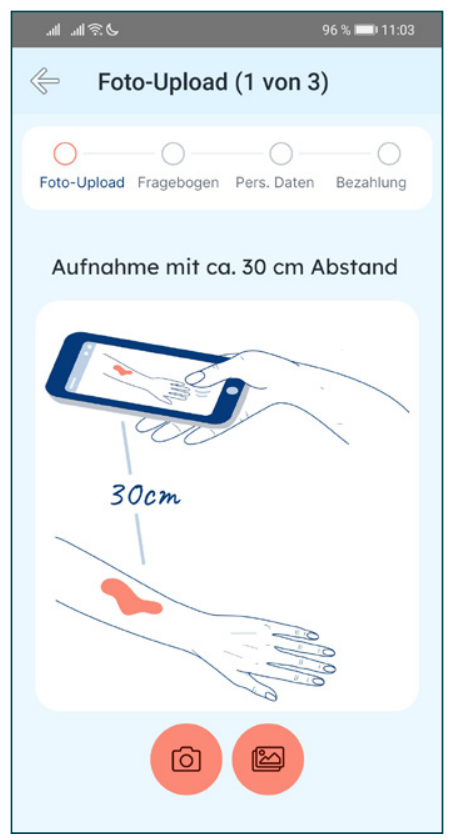

Per Foto und App zur Diagnose

\section{Dem Patienten viel Zeit sparen}

Einen anderen Ansatz der digitalen Dermatologie präsentierte Jeremy Henrichs. Er war selbst als Kind/Jugendlicher Betroffener einer Neurodermitis und ist auch deshalb Mitbegründer und Geschäftsführer (CEO) von NALA, einer App für Menschen, die an dieser Erkrankung leiden.

Die gesamte Patientenreise deutlich abgekürzt werden, benennt Henrichs den Sinn dieser App. Digitale Tagebücher zum Beispiel könnten viel schneller Aufschluss darüber bringen, was an Nahrung, sollte digitalisiert, und damit
Cremes oder Allergenen einen Trigger der Erkrankung darstellt. «Dies ist gerade bei einer so multifaktoriellen Erkrankung wie der Neurodermitis sehr hilfreich», betont Henrichs. Praktisch läuft dies folgendermaßen ab: sobald die Diagnose Neurodermitis feststeht, kann man sich die App herunterladen. Darauf finden sich 3-minütige Workshops zur Behandlung der Haut, ferner können Daten zur eigenen Haut aufgezeichnet werden. Dies kann in Form von Bildern geschehen oder mittels Aufzeichnungen in den verschiedenen Tagebüchern.

In einer Empfehlungsmaschine von NALA werden die Daten eingespeist, und auf dieser Basis individualisierte Behandlungsvorschläge generiert. Dieser Kreislauf kann immer wieder durchlaufen und aktualisiert werden. Erstellt werden können daraus Patient Reported Outcomes (PRO), welche die Lebensqualität abbilden. Und schließlich ist mit einem Klick ein PDF-Report abrufbar, der in Form einer Dokumentation mit dem Arzt besprochen werden kann. Im Hintergrund der Empfehlungen für den Patienten steht ein umfangreiches Team von Experten, wie etwa Ärzten verschiedener Fachrichtungen, Psychologen, Ernährungswissenschaftler usw., welche die Empfehlungsmaschine mit neuesten wissenschaftlichen Erkenntnissen updaten. Soweit möglich kommt dabei auch KI zum Einsatz. Das generelle Ziel einer solchen Form der Digitalisierung ist der viel schnellere und häufigere Umlauf von Maßnahmen zur Verbesserung der Haut und einem Feedback, also der Reaktion hinsichtlich des Erfolgs oder Misserfolgs der jeweiligen Maßnahme. Dabei kann es sich um direkte Anwendungen handeln, oder ebenso um Vermeidungen von Triggern in Nahrung, Umwelt oder psychologischen Belastungen. «Unser Ziel ist es, die Zeit von Diagnose bis zu einer glücklichen Haut von vielen Jahren/Jahrzehnten auf wenige Monate abzukürzen», fasst Henrichs zusammen.

\section{$\mathrm{KI}$ vereinfacht Diagnose und Verlaufskontrolle}

Prof. Dr. med. Dr. sc. nat. Alexander Navarini, Universitätsspital Basel, begann seinen Vortrag mit dem aktuellen «Optimum der digitalen Dermatologie», nämlich der Ganzkörper-Fotografie. Mit zahlreichen Aufnahmen in kürzester Zeit findet ein Screening statt. Darin implementiert ist ein Programm mit künstlicher Intelligenz (KI), welches die Bilder analysiert und erkennt, ob es sich um Muttermale, Warzen, Blutschwämmchen oder auch Malignitäten handelt. «Wir haben dieses Gerät seit 2019 in der Dermatologie des Universitäts Spitals Basel verfügbar.»

Der Vorteil daran ist, erläutert der Dermatologe, dass in einer Nachkontrolle - etwa nach einem halben oder einem Jahr - dieses Gerät exakt Veränderungen darstellen kann. «Diese Form der künstlichen Intelligenz kann eine erhebliche Hilfestellung für den Dermatologen darstellen», meint Navarini.

Dabei geht es zunächst um eine Klassifikation, also die Unterscheidung, ob es sich um eine Malignität oder etwa ein Naevus handelt. Des Weiteren geht es um Segmentierung, erklärt der Hautarzt. So 


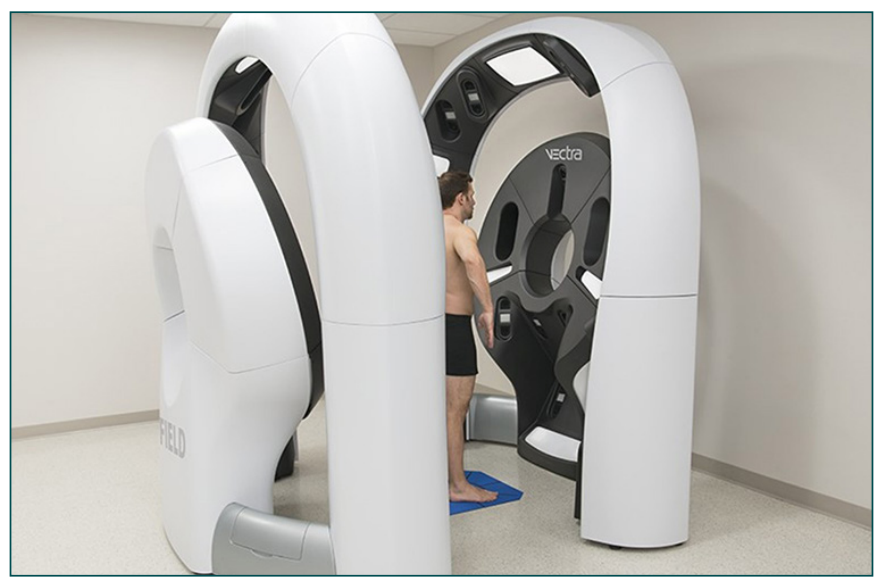

Ganzkörper-Fotografie: Das Optimum der digitalen Dermatologie

messen diese Geräte Flächen einer Dermatose, etwa die betroffenen Areale einer Psoriasis; oder das System zählt die Pusteln bei der Psoriasis pustulosa. Dies spart Zeit und dient der Verlaufskontrolle. Außerdem ist diese Form der KI in der Lage prädiktiv zu sagen, welches Outcome zu erwarten ist.

\section{Möglichkeiten und Limitierungen von Dr. Google}

Ein gänzlich anderes Thema ist der Einsatz von KI bei entsprechenden Anwendungen von großen IT-Unternehmen wie Google. Noch ist nicht klar, was solche Firmen wirklich anbieten wollen und können - oder auch dürfen, gibt Navarini zu bedenken. «Tatsächlich hat in den USA die Tele-Dermatologie um 48\% in den letzten fünf Jahren zugenommen», hält er fest. In Europa ist der Trend ähnlich aber etwas verzögert.

Mittlerweile werden viele Apps zur Selbstdiagnose von Hautkrebs angeboten. Diese sind allerdings teilweise scharf kritisiert worden - «und das wohl zu Recht», wie der Hautarzt meint.

Möglicherweise werden - als Kompromiss - solche autonomen Apps auf den Markt gebracht, die bei Einsenden von Bildern lediglich potenzielle Differenzial-Diagnosen anbieten. Inwieweit dies von den Nutzern jedoch akzeptiert wird, wenn etwa auch die Differenzial-Diagnose Malignom dabei ist, bleibt abzuwarten, meint
Navarini. Die Vision einer modernen Dermatologie, schaut er in die Zukunft, sieht eine Bildgebung bei jeder Visite beim Hautarzt vor. Der Dermatologe hat automatisch einen visuellen Vergleich mit den Vorbefunden. Bereits bei der Erstvorstellung wird er von KI unterstützt, die Vorschläge zur Diagnostik macht und Empfehlungen zur Therapie ausspricht. Quantitative Messungen werden erheblich erleichtert und deutlich schneller prozessiert.

\section{Steht ein Arzt hinter der Maschine - auch eine Frage der Ethik}

Sowohl die Dr Lang in Bezug auf die bei dermanostic stattfindende Telemedizin als auch Prof. Navarini wiesen jedoch darauf hin, dass bei ihnen hinter jeder Diagnose und letztlich auch Therapieempfehlung ein Facharzt steht, der im persönlichen Gespräch die Behandlung - sofern nötig - fortsetzen kann. Dies mag auch eine wichtige Rolle spielen bei der Frage, wer im Ernstfall (z.B. einer falsch-negativen Diagnose) die Verantwortung trägt, und zwar sowohl im juristischen, aber auch ethischen Sinne.

Prof. Dr. Stefan Heinemann lehrt Wirtschaftsethik an der FOM Hochschule in Essen. Er konzediert der digitalen Dermatologie ein beträchtliches Potenzial in ihrer unterstützenden Funktion. Sie kann dem Hautarzt vielfältige Aufgaben abnehmen oder erleichtern. Sie kann auch dort Lücken füllen, wo eine persönliche Betreuung durch den Dermatologen - zumindest nicht zeitnah - zur Verfügung steht.

So kann die digitale Medizin für eine Verbesserung von Diagnostik, Therapie und Nachsorge, und insbesondere im Bereich der Prävention genutzt werden. Dabei darf aber die conditio humana nicht aus dem Blick verloren werden. Er betont ausdrücklich: Ein reiner Algorithmus kann keine moralische Verantwortung übernehmen. Ebenso wenig kann er Empathie und Einfühlungsvermögen vermitteln. Dies kann allein der Arzt als Mensch. Wo der Mensch als Patient mit seinem Anliegen von Heilung und Nähe nicht mehr im Zentrum steht, wird sich ein vordergründiger Nutzen durch Digitalisierung aber auch sehr schnell abnutzen.

Reimund Freye, Baden-Baden 